\title{
TOTALLY UMBILICAL SUBMANIFOLDS OF QUATERNION-SPACE-FORMS
}

\author{
BANG-YEN CHEN
}

(Received 1 August 1977)

Communicated by J. Virsik

\begin{abstract}
Totally umbilical submanifolds of dimension greater than four in quaternion-space-forms are completely classified.
\end{abstract}

Subject classification (Amer. Math. Soc. (MOS) 1970): 53 B 25, 53 C 40.

\section{Introdaction}

A quaternion manifold is defined as a Riemannian manifold whose holonomy group is a subgroup of $\mathrm{Sp}(m) \cdot \mathrm{Sp}(1)=\mathrm{Sp}(m) \times \mathrm{Sp}(1) /\{ \pm$ identity $\}$. The irreducible symmetric spaces $\mathrm{Sp}(1+m) / \mathrm{Sp}(1) \times \mathrm{Sp}(m)$ and $\mathrm{Sp}(1, m) / \mathrm{Sp}(1) \times \mathrm{Sp}(m)$ are the two most important examples of quaternion manifolds. It is well known that these two spaces have constant quaternion sectional curvature for $m$ greater than or equal to 2 . We simply call quaternion manifolds with constant quaternion sectional curvature the quaternion-space-forms.

In this paper, we shall completely classify totally umbilical submanifolds of dimension greater than 4 in quaternion-space-forms. The dimension of a manifold will always indicate its real dimension. We shall prove the following

THEOREM. Let $N$ be an $n$-dimensional $(n>4)$ totally umbilical submanifold in a $4 m$-dimensional quaternion-space-form $M$ of quaternion sectional curvature $c \neq 0$. Then $N$ is one of the following submanifolds:

(a) a quaternion-space-form immersed in $M$ as a totally geodesic, quaternion submanifold, or

Partially supported by NSF under Grant MCS 76-06318. 
(b) a complex-space-form immersed in $M$ as a totally geodesic, half-quaternion submanifold, or

(c) a real-space-form immersed in $M$ as a totally geodesic, totally real submanifold, or

(d) a real-space-form immersed in $M$ as a totally real extrinsic sphere.

Case (b) (respectively, (c) and (d)) occurs only when $n \leqslant 2 m$ (respectively, $n \leqslant m$ and $n<m)$.

Since every $4 m$-dimensional quaternion-space-form of zero quaternion sectional curvature is a flat space, classification of totally umbilical submanifolds in such a space follows from a well-known result of Cartan (1946). For the classifications of totally umbilical submanifolds in a complex-space-form and Cayley plane, see Chen-Ogiue (1974) and Chen (1977), respectively.

\section{Preliminaries}

Let $M$ be a $4 m$-dimensional quaternion manifold with metric $g$. There exists a 3-dimensional vector space $V$ of tensors of type $(1,1)$ with local basis of almost Hermitian structures $I, J, K$ such that (i) $I J=-J I=K$, and (ii) for any local cross-section $\psi$ of $V, \nabla_{X} \psi$ is also a cross -section of $V$, where $X$ is an arbitrary vector field in $M$ and $\nabla$ the Levi-Civita connection on $M$. It is well known that the existence of such vector bundle $V$ on a Riemannian manifold implies that it is a quaternion manifold (Ishihara, 1974).

Let $X$ be a unit vector on the quaternion manifold $M$. Then $X, I X, J X$ and $K X$ form an orthonormal frame in $M$. We denote by $Q(X)$ the 4-plane spanned by them, and call it the quaternion 4-plane determined by $X$. Every 2-plane (or simply plane) in a quaternion 4-plane is called a quaternion plane. The sectional curvature for a quaternion plane is called a quaternion sectional curvature. A quaternion manifold is called a quaternion-space-form if its quaternion sectional curvatures are equal to a constant $c$. It is known that a quaternion manifold is a quaternion-space-form if and only if its curvature tensor $R$ is of the following form:

$$
\begin{array}{rl}
R(X, Y) Z=1 & c\{g(Y, Z) X-g(X, Z) Y+g(I Y, Z) I X-g(I X, Z) I Y \\
+ & 2 g(X, I Y) I Z+g(J Y, Z) J X-g(J X, Z) J Y \\
& +2 g(X, J Y) J Z+g(K Y, Z) K X-g(K X, Z) K Y \\
& +2 g(X, K Y) K Z\}
\end{array}
$$

for some constant $c$. Moreover, it is known that quaternion-space-forms are locally symmetric (Ishihara, 1974).

For any two vectors $X, Y$ in $M$, if $Q(X)$ and $Q(Y)$ are orthogonal, the plane $\pi(X, Y)$ spanned by $X, Y$ is said to be totally real. An isometric immersion $x: N \rightarrow M$ 
from a Riemannian manifold $N$ into $M$ is said to be totally real if each tangent plane of $N$ is carried into a totally real plane by $x$ in $M$.

Let $N^{\prime}$ be an almost Hermitian manifold and $y: N^{\prime} \rightarrow M$ an isometric immersion form $N^{\prime}$ into $M$. Then $N^{\prime}$ is called a half-quaternion submanifold of $M$ if each holomorphic plane in $N^{\prime}$ is carried into a quaternion plane in $M$. A Riemannian manifold is called a real-space-form if it has constant sectional curvature and a Kaehler manifold is called a complex-space-form if it has constant holomorphic sectional curvature.

Let $M^{\prime}$ be a quaternion manifold and $z: M^{\prime} \rightarrow M$ an isometric immersion from $M^{\prime}$ into $M$. We call $M^{\prime}$ a quaternion submanifold of $M$ if quaternion 4-planes in $M^{\prime}$ are carried into quaternion 4-planes by $z$.

\section{Basic formulas}

Let $N$ be an $n$-dimensional submanifold of a quaternion manifold $M$ with metric $g$ and local almost Hermitian structures $I, J, K$. We have

$$
\begin{aligned}
& I^{2}=J^{2}=K^{2}=-1, \\
& I J=-J I=K, \quad J K=-K J=I, \quad K I=-I K=J, \\
& g(I X, I Y)=g(J X, J Y)=g(K X, K Y)=g(X, Y) .
\end{aligned}
$$

Moreover, for any two orthonormal vectors $X, Y$ in $M$ which span a totally real plane, we have

$$
g(\psi X, \rho Y)=0, \quad \psi, \rho=I, J, \text { or } K .
$$

Let $\nabla$ and $\nabla^{\prime}$ be the Levi-Civita connections on $M$ and $N$, respectively. The second fundamental form $h$ of the immersion is defined by the equation; $h(X, Y)=\nabla_{X} Y-\nabla_{X}^{\prime} Y$ for vector fields $X, Y$ tangent to $N$. For a vector field $\xi$ normal to $N$, we write

$$
\nabla_{X} \xi=-A_{\xi} X+D_{X} \xi
$$

where $-A_{\xi} X$ (respectively, $D_{X} \xi$ ) denotes the tangential component (respectively, the normal component) of $\nabla_{X} \xi$. A normal vector field $\xi$ is said to be parallel if $D \xi=0$. The submanifold $N$ is said to be totally umbilical if $h(X, Y)=g(X, Y) H$, for all vector fields $X, Y$ tangent to $N$, where $H=$ trace $h / n$ is the mean curvature vector of $N$ in $M$. If the second fundamental form $h$ vanishes identically, $N$ is called a totally geodesic submanifold of $M$. A totally umbilical submanifold with nonzero parallel mean curvature vector is called an extrinsic sphere.

Let $R, R^{\prime}$ and $R^{N}$ be the curvature tensors associated with $\nabla, \nabla^{\prime}$ and $D$, respectively. For the second fundamental form $h$ of $N$ in $M$, we define the covariant 
derivative, denoted by $\nabla_{X} h$, to be

$$
\left(\nabla_{X} h\right)(Y, Z)=D_{X}(h(Y, Z))-h\left(\nabla_{X}^{\prime} Y, Z\right)-h\left(Y, \nabla_{X}^{\prime} Z\right)
$$

Then, for all vector fields $X, Y, Z, W$ tangent to $N$ and vector fields $\xi, \eta$ normal to $N$, the equations of Gauss, Codazzi and Ricci take the following forms (see Chen, 1973):

$$
\begin{gathered}
g(R(X, Y) Z, W)=g\left(R^{\prime}(X, Y) Z, W\right)+g(h(X, Z), h(Y, W)) \\
-g(h(X, W), h(Y, Z)),
\end{gathered}
$$

where $\perp$ in (3.8) denotes the normal component.

We call the submanifold $N$ an invariant submanifold of $M$ if we have

$$
R(X, Y) T N \subseteq T N
$$

for all $X, Y$ in the tangent bundle $T N$ of $N$.

\section{Proof of the theorem}

We first prove the following lemmas.

LEMMA 1. Under the hypothesis of the theorem, $N$ is an invariant submanifold and $H$ is parallel.

Proof. Since $N$ is a totally umbilical submanifold of dimension greater than 4 in a quaternion-space-form $M$, we have

$$
h(X, Y)=g(X, Y) H
$$

for all vector fields $X, Y$ tangent to $N$. By (3.6), we have

$$
\left(\nabla_{X} h\right)(Y, Z)=g(Y, Z) D_{X} H \text {. }
$$

Thus, equation (3.8) reduces to

$$
(R(X, Y) Z)^{\perp}=g(Y, Z) D_{X} H-g(X, Z) D_{Y} H .
$$

Since the dimension of $N$ is greater than 4, for each unit vector field $X$ tangent to $N$, there is a unit vector field $Y$ tangent to $N$ orthogonal to $Q(X)$. For such $Y$, (4.3) gives $(R(X, Y) Y)^{\perp}=D_{X} H$. On the other hand, (2.1) and (3.4) imply $(R(X, Y) Y)^{\perp}=0$. Thus, $D_{X} H=0$ for all vectors $X$ tangent to $N$, that is, $H$ is 
parallel. Substituting this into (4.3), we see that $(R(X, Y) Z)^{\perp}=0$, for all vector fields $X, Y, Z$ tangent to $N$. Thus, $N$ is an invariant submanifold of $M$.

LeMma 2. Under the hypothesis of the theorem, $N$ is locally symmetric.

Proof. Since $H$ is parallel by Lemma 1, (4.2) gives $\nabla h=0$. From (3.7) and (3.8), we find

$$
\begin{array}{rlr}
g(R(X, Y) Z, W) & =g\left(R^{\prime}(X, Y) Z, W\right)+\alpha^{2}\{g(X, Z) g(Y, W) \\
& g(R(X, Y) Z, \xi)=0, & -g(X, W) g(Y, Z)\} .
\end{array}
$$

for all vector fields $X, Y, Z, W$ tangent to $N$ and vector field $\xi$ normal to $N$, where $\alpha^{2}=g(H, H)$. From (4.4) and (4.5), we get

$$
R^{\prime}(X, Y) Z=R(X, Y) Z+\alpha^{2}\{g(Y, Z) X-g(X, Z) Y\} .
$$

Because $H$ is parallel, $\alpha$ is a constant. Thus, by using $\nabla R=0$, we get

$$
\begin{aligned}
\nabla_{U}(R(X, Y) Z)= & R\left(\nabla_{U} X, Y\right) Z+R\left(X, \nabla_{U} Y\right) Z+R(X, Y) \nabla_{U} Z \\
= & R\left(\nabla_{U}^{\prime} X, Y\right) Z+R\left(X, \nabla_{U}^{\prime} Y\right) Z+R(X, Y) \nabla_{U}^{\prime} Z \\
& +R(h(X, U), Y) Z+R(X, h(U, Y)) Z \\
& +R(X, Y)(h(U, Z)) .
\end{aligned}
$$

Consequently, (4.5) and (4.7) imply

$$
\begin{aligned}
& U(g(R(X, Y) Z, W))= g( \\
&\left.R\left(\nabla_{U}^{\prime} X, Y\right) Z, W\right)+g\left(R\left(X, \nabla_{U}^{\prime} Y\right) Z, W\right) \\
&+ g\left(R(X, Y) \nabla_{U}^{\prime} Z, W\right)+g\left(R(X, Y) Z, \nabla_{U}^{\prime} W\right),
\end{aligned}
$$

for all vector fields $X, Y, Z, W, U$ tangent to $N$. From (4.6) and (4.8), we find

$$
\begin{aligned}
U(g(R(X, Y) Z, W))=U( & \left.\left(R^{\prime}(X, Y) Z, W\right)\right)-g\left(\left(\nabla_{U}^{\prime} R^{\prime}\right)(X, Y) Z, W\right) \\
+ & +\alpha^{2} U\{g(X, Z) g(Y, W)-g(X, W) g(Y, Z)\} .
\end{aligned}
$$

Therefore, (4.4) and (4.9) imply that $N$ is locally symmetric.

LEMMA 3. If $N$ is an $\mathrm{n}$-dimensional $(n \geqslant 4)$ invariant submanifold of a quaternionspace-form $M$ of quaternion sectional curvature $c \neq 0$, then $N$ is a totally real submanifold, or a half-quaternion submanifold, or a quaternion submanifold of $M$.

Proof. Let $X, Y$ be two vectors tangent to $N$. From (2.1) we find

$$
\begin{aligned}
R(X, Y) X=\frac{1}{4} c\{g(Y, X) X- & g(X, X) Y+3 g(I Y, X) I X \\
& +3 g(J Y, X) J X+3 g(K Y, X) K X\} .
\end{aligned}
$$


Since $N$ is an invariant submanifold of $M,(4.10)$ implies

$$
g(Y, I X) I X+g(Y, J X) J X+g(Y, K X) K X \in T N,
$$

from which we see that if the component of $Y$ in $Q(X)$ is nonzero, the component of $Y$ in $Q(X)$ is nonzero, the component lies in $T N$. Therefore, we may choose an orthonormal basis for each $T_{p} N, p \in N$, in the following form:

$$
e_{1}, \ldots, e_{i_{1}}, e_{i_{1}+1}, \ldots, e_{i_{1}+i_{2}}, \ldots, e_{i_{1}+\ldots+i_{k-1}+1}, \ldots, e_{i_{1}+\cdots+i_{k}},
$$

where each $B_{l}=\left\{e_{i_{1}+\ldots+i_{l-1}+1}, \ldots, e_{i_{1}+\ldots+i_{l}}\right\}$ is a subset of a quaternion 4-plane $Q\left(E_{l}\right), l=1, \ldots, k$, for some $E_{l}$; moreover, $Q\left(E_{1}\right), \ldots, Q\left(E_{k}\right)$ are mutually orthogonal. In particular, the number of elements in each $B_{l}$ is fixed as $p$ varies on $N$.

Case (1). If each $B_{l}$ contains exactly one element, then $N$ is a totally real submanifold of $M$.

Case (2). If each $B_{l}$ contains exactly four elements, then each $B_{l}$ spans a quaternion 4-plane. The quaternion structure on $M$ then induces a canonical quaternion structure on $N$. With respect to this canonical quaternion structure, $N$ becomes a quaternion submanifold of $M$.

Case (3). If neither case (1) nor case (2) holds, then there is a $B_{j}$ which contains either 2 or 3 elements. Without loss of generality, we may assume that $l=1$.

Case (3.1). If $B_{1}=\left\{e_{1}, e_{2}\right\}$, then, for any $e \in B_{l}, l \neq 1$, we have

$$
R\left(e_{1}, e_{2}\right) e=\frac{1}{2} c\left\{g\left(e_{1}, I e_{2}\right) I e+g\left(e_{1}, J e_{2}\right) J e+g\left(e_{1}, K e_{2}\right) K e\right\}
$$

by virtue of (2.1). Since $g\left(e_{1}, e_{2}\right)=0$ and $e_{1}, e_{2}$ lie in the same quaternion 4-plane, at least one of $g\left(e_{1}, I e_{2}\right), g\left(e_{1}, J e_{2}\right)$ and $g\left(e_{1}, K e_{2}\right)$ is nonzero. Thus, from (3.10), $B_{1}$ contains at least two elements. This shows that if $B_{1}$ contains two elements, all other $B_{l}$ contain at least two elements. If each $B_{l}$ contains exactly two elements, then we have

$$
B_{1}=\left\{e_{1}, e_{2}\right\}, \quad B_{2}=\left\{e_{3}, e_{4}\right\}, \ldots, B_{k}=\left\{e_{2 k-1}, e_{2 k}\right\}, \quad 2 k=n .
$$

Since $e_{2 i-1}$ and $e_{2 i}$ are two orthonormal vectors lying in the same quaternion 4-plane, $e_{2 i}$ is a linear combination of $I e_{2 i-1}, J e_{2 i-1}, K e_{2 i-1}$, namely,

$$
e_{2 i}=a I e_{2 i-1}+b J e_{2 i-1}+c K e_{2 i-1} \text {, }
$$

with $a^{2}+b^{2}+c^{2}=1$. Now, we define a $(1,1)$-tensor $j$ on $T N$ by

$$
=a I+b J+c K,
$$

then $j^{2}=-1$, that is, $j$ defines an almost complex structure on $N$. It is clear that $N$ with this canonical almost complex structure forms a half-quaternion submanifold of $M$. Moreover, $j$ with the induced metric is almost Hermitian. 
Case (3.2). If $B_{1}=\left\{e_{1}, e_{2}, e_{3}\right\}$, then, for any $e \in B_{l}, l \neq 1$, we have

$$
R\left(e_{\alpha}, e_{\beta}\right) e=\frac{1}{2} c\left\{g\left(e_{\alpha}, I e_{\beta}\right) I e+g\left(e_{\alpha}, J e_{\beta}\right) J e+g\left(e_{\alpha}, K e_{\beta}\right) K e\right\}
$$

for all $\alpha, \beta=1,2,3$. Since $N$ is an invariant submanifold, $R\left(e_{\alpha}, e_{\beta}\right) e \in T N$. Because the following matrix

$$
\left(\begin{array}{lll}
g\left(e_{1}, I e_{2}\right) & g\left(e_{1}, J e_{2}\right) & g\left(e_{1}, K e_{2}\right) \\
g\left(e_{1}, I e_{3}\right) & g\left(e_{1}, J e_{3}\right) & g\left(e_{1}, K e_{3}\right) \\
g\left(e_{2}, I e_{3}\right) & g\left(e_{2}, J e_{3}\right) & g\left(e_{2}, K e_{3}\right)
\end{array}\right)
$$

is of rank 3, (4.12) implies that $I e, J e$ and $K e$ lie in $T N$. Thus, $B_{l}$ contains 4 elements. Applying the same argument to $B_{1}$, we obtain a contradiction.

LEMMA 4. If $N$ is a quaternion submanifold of a quaternion manifold $M$, then $N$ is totally geodesic.

Proof. From the definition of the second fundamental form, we have

$$
h(X, I Y)=\nabla_{X}(I Y)-\nabla_{X}^{\prime}(I Y)=I \nabla_{X} Y-\nabla_{X}^{\prime}(I Y)+\left(\nabla_{X} I\right) Y .
$$

Since $\nabla_{X} I$ is a linear combination of $I, J, K,\left(\nabla_{X} I\right) Y$ is tangent to $N$. Comparing the normal components of both sides of (4.13), we find

$$
h(X, I Y)=\operatorname{Ih}(X, Y)
$$

Hence, we get

$$
h(I X, I Y)=-h(X, Y)
$$

Similarly, we have

$$
h(J X, J Y)=h(K X, K Y)=-h(X, Y) .
$$

On the other hand, by using $K=I J$, we find $h(K X, K Y)=-h(J X, J Y)=h(X, Y)$. Comparing this with (4.16), we get $h(X, Y)=0$. Thus, $N$ is totally geodesic in $M$.

LEMMA 5. Let $N$ be a totally umbilical submanifold of dimension $n(n>4)$ in $a$ quaternion-space-form of quaternion sectional curvature $c \neq 0$. If $N$ is a half-quaternion submanifold, then $N$ is a complex-space-form immersed in $M$ as a totally geodesic submanifold in $M$.

Proof. If $N$ is a half-quaternion submanifold of $M, N$ has a canonical almost Hermitian structure $(j, g)$. From Lemma $2, N$ is locally symmetric with respect to the induced metric, thus, $M$ is Kaehlerian. From the equation of Gauss, for any 
plane section $\pi$ in $N$, the sectional curvature $\sigma$ and $\sigma^{\prime}$ of $\pi$ in $M$ and $N$ satisfy $\sigma^{\prime}(\pi)=\sigma(\pi)+\alpha^{2}$, where $\alpha^{2}=g(H, H)$. Since, for any holomorphic plane section $\pi(X, j X)$, the sectional curvature of the quaternion-space-form $M$ satisfies $\sigma(\pi(X, j X))=c$, the holomorphic sectional curvature of $N$ is equal to the constant $c+\alpha^{2}$. Thus, $N$ is a complex-space-form. Therefore, $N$ as well as $M$ are 1 -pinched. Consequently, the ratio of $c+\alpha^{2}: 1 c+\alpha^{2}$ is $4: 1$. But this is impossible unless $\alpha=0$, that is, $N$ is minimal. Since $N$ is totally umbilical, $N$ is totally geodesic.

Now, we return to the proof of the theorem. By Lemma $1, N$ is invariant. Thus, Lemma 3 implies that $N$ is a totally real submanifold, or a half-quaternion submanifold, or a quaternion submanifold.

If $N$ is a quaternion submanifold, Lemma 4 implies that $N$ is totally geodesic. Since the quaternion structure on $N$ is obtained from the restriction of the quaternion structure on $M$, equation (3.7) of Gauss implies that the curvature tensor of $N$ is also given in the form of (2.1). Thus $N$ is also a quaternion-space-form.

If $N$ is a half-quaternion submanifold, Lemma 5 implies that $N$ is a complexspace-form immersed in $M$ as a totally geodesic submanifold.

If $N$ is a totally real submanifold, then, for any orthonormal vectors $X, Y$ tangent to $N$, the quaternion 4-planes $Q(X)$ and $Q(Y)$ are orthogonal. Thus, (2.1) gives $\sigma(\pi(X, Y))=\frac{1}{1} c$. By the equation of Gauss, $N$ is a real-space-form of sectional curvature $1 c+\alpha^{2}$. If $N$ is not totally geodesic in $M, H \neq 0$. By the parallelism of $H$, we find from the equation of Ricci that

$$
g(R(X, Y) H, I Y)=0,
$$

for any orthonormal vectors $X, Y$ tangent to $N$. On the other hand, for such $X$ and $Y$, equation (2.1) gives

$$
g(R(X, Y) H, I Y)=-\frac{1}{c} g(I X, H) g(I Y, I Y) .
$$

Thus, $g(I X, H)=0$. Similarly, we have $g(J X, H)=g(K X, H)=0$. Since this is true for all $X$ tangent to $N, H$ is perpendicular to $Q(T N)$. Thus, $m>n=\operatorname{dim} N$. This completes the proof of the theorem.

\section{Remarks}

Remark 1. If $n=3$, Lemma 3 is false. This follows from the fact that for any 3-dimensional subspace $\pi$ of a quaternion 4-plane $Q(X)$ in a quaternion-spaceform, there exists a totally geodesic submanifold in $M$ which is tangent to $\pi$.

REMARK 2. Let $M$ be the complete quaternion-space-form

$$
\mathrm{Sp}(1+m) / \mathrm{Sp}(1) \times \mathrm{Sp}(m),
$$


and let $N$ be the real-space-form $\mathrm{SO}(1+m) /\{ \pm 1\} \times \mathrm{SO}(m)$. Then, by regarding $\mathrm{SO}(1+m)$ as a subgroup of $\mathrm{Sp}(1+m)$ in the natural way, it is clear that $\mathrm{SO}(1+m) \cap \mathrm{Sp}(1) \times \mathrm{Sp}(m)=\{ \pm 1\} \times \mathrm{SO}(m)$. This shows that

$$
\mathrm{SO}(1+m) /\{ \pm 1\} \times \operatorname{SO}(m)
$$

can be imbedded in $\mathrm{Sp}(1+m) / \mathrm{Sp}(1) \times \mathrm{Sp}(m)$ as a totally geodesic submanifold. Moreover, it is clear that the imbedding is totally real.

Since $\mathrm{SO}(m) /\{1\} \times \mathrm{SO}(m-1)$ can be imbedded in $\mathrm{SO}(m+1) /\{ \pm 1\} \times \mathrm{SO}(m)$ as an extrinsic sphere, there is an $(m-1)$-dimensional real-space-form imbedded in a $4 m$-dimensional quaternion-space-form as an extrinsic sphere. Thus, both cases (c) and (d) happen.

ReMARK 3. Since $S U(1+m) / S\left(U_{1} \times U_{m}\right)$ can be imbedded in

$$
\mathrm{Sp}(1+m) / \mathrm{Sp}(1) \times \mathrm{Sp}(m)
$$

as a totally geodesic, half-quaternion submanifold in a natural way, case (b) of the theorem occurs.

Remark 4. Since $\mathrm{Sp}(1+q) / \mathrm{Sp}(1) \times \mathrm{Sp}(q)$ can be imbedded in

$$
\mathrm{Sp}(1+m) / \mathrm{Sp}(1) \times \mathrm{Sp}(m)
$$

as a totally geodesic, quaternion submanifold in a natural way for $q<m$, case (a) of the theorem occurs.

REMARK 5. If we consider the corresponding results in Remarks 2,3 and 4 for spaces of non-compact type, we see that cases (a)-(d) in the theorem occur for $c<0$ as well as $c>0$.

\section{References}

E. Cartan (1946), Leçons sur la Géométrie des Espaces de Riemann (Gauthier-Villars, Paris, 2nd ed.).

B. Y. Chen (1973), Geometry of Submanifolds (M. Dekkers, New York).

B. Y. Chen (1977), "Totally umbilical submanifolds of Cayley plane", Soochow J. Math. Natur. Sci., 3, 1-7.

B. Y. Chen and K. Ogiue (1974), "Two theorems on Kaehler manifolds", Michigan Math. J. 21, 225-229.

S. Ishihara (1974), “Quaternion Kaehlerian manifolds", J. Differential Geometry 9, 483-500.

Department of Mathematics

Michigan State University

East Lansing, MI 48824 